\title{
High-quality two-dimensional electron gas in undoped InSb quantum wells
}

\author{
Zijin Lei $\odot,{ }^{1, *}$ Erik Cheah, ${ }^{1}$ Km Rubi $\odot,{ }^{2, \dagger}$ Maurice E. Bal, ${ }^{2}$ Christoph Adam $\odot,{ }^{1}$ Rüdiger Schott $\odot,{ }^{1}$ Uli Zeitler $\odot,{ }^{2}$ \\ Werner Wegscheider, ${ }^{1}$ Thomas Ihn $\odot,{ }^{1,3}$ and Klaus Ensslin $\oplus^{1,3}$ \\ ${ }^{1}$ Solid State Physics Laboratory, ETH Zurich, CH-8093 Zurich, Switzerland \\ ${ }^{2}$ High Field Magnet Laboratory (HFML-EMFL), Radboud University, 6525 ED Nijmegen, the Netherlands \\ ${ }^{3}$ ETH Zurich Quantum Center, CH-8093 Zurich, Switzerland
}

(Received 22 October 2021; accepted 29 December 2021; published 19 January 2022)

\begin{abstract}
We report on transport experiments through high-mobility gate-tunable undoped InSb quantum wells (QWs). Due to the elimination of any Si modulation doping, the gate-defined two-dimensional electron gases in the QWs display a significantly increased mobility of $260000 \mathrm{~cm}^{2} / \mathrm{Vs}$ at a rather low density of $2.4 \times 10^{11} \mathrm{~cm}^{-2}$. Using magnetotransport experiments, we characterize spin-orbit interactions by measuring weak antilocalization. Furthermore, by measuring Shubnikov-de Haas oscillations in tilted magnetic fields, we find that the $g$ factor agrees with $\mathbf{k} \cdot \mathbf{p}$ theory calculations at low magnetic fields but grows with spin polarization and carrier density at high magnetic fields. Additionally, signatures of Ising quantum Hall ferromagnetism are found at filling factor $v=2$ for tilt angles where the Landau level energy equals the Zeeman energy. Despite the high mobility, the undoped InSb QWs exhibit no fractional quantum Hall effect up to magnetic fields of $25 \mathrm{~T}$.
\end{abstract}

DOI: 10.1103/PhysRevResearch.4.013039

\section{INTRODUCTION}

InSb is a narrow-gap III-V compound known for its light effective mass, large $g$ factor in bulk material, and strong spinorbit interactions (SOIs) [1-5]. These unique properties are interesting for potential applications such as high-frequency electronics [1], optoelectronics [6], and spintronics [7]. Recently, InSb, together with InAs, has attracted attention as a potential platform for topological quantum information processing [8]. As introduced in a series of both theoretical and experimental works, a topological nontrivial phase can be achieved in a semiconductor-superconductor hybridized device. It needs a sophisticated control of Zeeman energy, chemical potential, Rashba SOI, and the phase of supercurrent if the system is two-dimensional (2D) to form a pair of Majorana zero modes on sample terminals [9-12]. Currently, experimental works on Majorana physics are primarily focused on InAs and InSb nanowires and InAs quantum wells (QWs), while the progress in InSb QWs is still hampered, and related publications are rare [11]. Although InSb QWs have been successfully grown using molecular beam epitaxy (MBE) methods [13], the technique to obtain high-quality 2D electron gases (2DEGs) in InSb QWs is still not as mature as that in InAs QWs. For instance, the mobility of InAs QWs has reached values as high as $\sim 2400000 \mathrm{~cm}^{2} / \mathrm{Vs}$, while this value is still $\sim 300000 \mathrm{~cm}^{2} / \mathrm{Vs}$ in InSb QWs [14-16]. In addi-

\footnotetext{
*Corresponding author: zilei@ phys.ethz.ch

${ }^{\dagger}$ Present address: National High Magnetic Field Laboratory, Los Alamos National Laboratory, Los Alamos, NM 87545, USA.

Published by the American Physical Society under the terms of the Creative Commons Attribution 4.0 International license. Further distribution of this work must maintain attribution to the author(s) and the published article's title, journal citation, and DOI.
}

tion, the carrier density in InSb QWs is not as stable as those in InAs and GaAs. A pinched-off channel always becomes conductive again after minutes when keeping gate voltages constant. Due to this shortcoming, nanoconstrictions based on InSb QWs still pose a number of challenges [17,18]. Recently, Kulesh et al. [19] presented a purely gate-defined quantum dot in an InSb QW. The instability of carrier density with time is best reduced by eliminating $\mathrm{Si}$ doping during the growth of the heterostructures. Then the carriers in these undoped InSb QWs are induced through an electric field applied by a global top gate. Though stable Coulomb blockade effects were achieved in their device [19], undoped InSb QWs need further optimization. As illustrated in the work of undoped $\mathrm{GaAs} / \mathrm{AlGaAs}$ heterostructures, the elimination of modulation doping minimizes remote ionized dopant scattering [20,21]. With the previous work in various III-V compound heterostructures as references, we expect that 2DEGs with both high mobility and stable density can be induced in undoped InSb QWs.

In this paper, we introduce undoped InSb QWs as a platform for magnetotransport experiments. Due to the elimination of modulation doping, gate-defined 2DEGs display a significantly increased mobility compared with modulationdoped devices at low carrier densities $<2.4 \times 10^{11} \mathrm{~cm}^{-2}$. Second, we manage to probe the tunable SOIs of the 2DEGs with the measurement of weak antilocalization (WAL). Furthermore, by coincidence measurements of the Shubnikov-de Haas $(\mathrm{SdH})$ oscillations in tilted magnetic fields, we find that the $g$ factor agrees with $\mathbf{k} \cdot \mathbf{p}$ theory calculations in low magnetic fields but grows with spin polarization and carrier density in high magnetic fields. Additionally, these 2DEGs show signatures of Ising quantum Hall ferromagnetism when the Zeeman energy equals the Landau energy in tilted magnetic fields. Finally, fractional quantum Hall effects (FQHEs) are still absent in high magnetic fields even in these high mobility devices. 


\section{SAMPLE PREPARATION AND MEASUREMENT METHODS}

The InSb QW sample we investigate here was grown on a (100) GaAs substrate by MBE. The growth process is based on a previous publication by Lehner et al. [13]. First, a specialized interfacial misfit transition to a GaSb buffer and an interlayer InAlSb buffer was employed to overcome the lattice mismatch between GaAs and InSb. The total thickness of the buffer system amounted to $\sim 3 \mu \mathrm{m}$. Then the 21-nm-thin InSb QW was surrounded by $\operatorname{In}_{0.9} \mathrm{Al}_{0.1} \mathrm{Sb}$ confinement barriers. On top of the QW, the thickness of the $\operatorname{In}_{0.9} \mathrm{Al}_{0.1} \mathrm{Sb}$ barrier was $50 \mathrm{~nm}$. The bottom barrier was a $2-\mu \mathrm{m}$-thick $\operatorname{In}_{0.9} \mathrm{Al}_{0.1} \mathrm{Sb}$ layer. There was no Si modulation doping performed during growth.

In contrast to the doped structure, the microfabrication of undoped InSb needs more optimization and additional steps and is presented in the following. First, a standard $400 \times$ $200 \mu \mathrm{m}$ Hall bar was defined using chemical etching with an etching depth of $\sim 80 \mathrm{~nm}$, deeper than the QW. Like the undoped GaAs/AlGaAs heterostructures, ohmic contacts are more challenging to achieve in undoped InSb QWs [20,21]. Here, layers of metals were evaporated after an Ar milling step to provide ohmic contacts. Because there were no carriers without an electric field applied, we needed to anneal the sample for the contact material to diffuse into the QWs both in the growth and the lateral direction. Therefore, the chance of avoiding an insulating gap between the alloyed contacts and 2DEGs was maximized. Ge/Ni/Au as contact metals were adopted because this alloy diffuses into III-V semiconductors even at relatively low temperatures. We also recommend a shallow $(\sim 10 \mathrm{~nm})$ etch on the areas where contacts locate to remove the oxidized layer on the surface and decrease the distance of metal diffusion to the QWs. In the next step, the sample was coated with a 40-nm-thick aluminum oxide (ALO) dielectric layer using atomic layer deposition (ALD) at a temperature of $150^{\circ} \mathrm{C}$ for $1.5 \mathrm{~h}$. These ALD parameters were optimized considering metal diffusion, dielectric layer quality, and preserving the mobility of 2DEGs. On the one hand, a longer heating time or higher temperature will reduce the mobility of the 2DEG, though the ALO layer will have better quality. On the other hand, the metal will not diffuse sufficiently if the heating is not enough. Finally, a Ti/Au top gate covering the Hall bar and the contacts was deposited by electron-beam evaporation. With this method, we successfully made 11 working devices from three nominally identical wafers. For all working samples, the contacts were measured to be ohmic, and the 2-terminal resistances of samples were within $10 \mathrm{k} \Omega$ when the carrier densities were maximum.

The transport measurements were accomplished using standard low-frequency lock-in techniques in a cryostat with a base temperature of $1.7 \mathrm{~K}$. First, with the top gates grounded, the QWs were completely insulating. We verified that a high-quality single-channel InSb 2DEG was formed when a positive top gate voltage $V_{\mathrm{TG}}$ was applied. Figure 1(a) shows the density $n$ determined via the classical Hall effect in small magnetic fields and the corresponding Drude mobility $\mu$ of the 2DEG as a function of $V_{\mathrm{TG}}$. This measurement was performed on a Hall bar oriented along the crystal direction $\left[\begin{array}{lll}1 & -1 & 0\end{array}\right]$. With the increase of $V_{\mathrm{TG}}, n$ increases up to $2.4 \times 10^{11} \mathrm{~cm}^{-2}$,
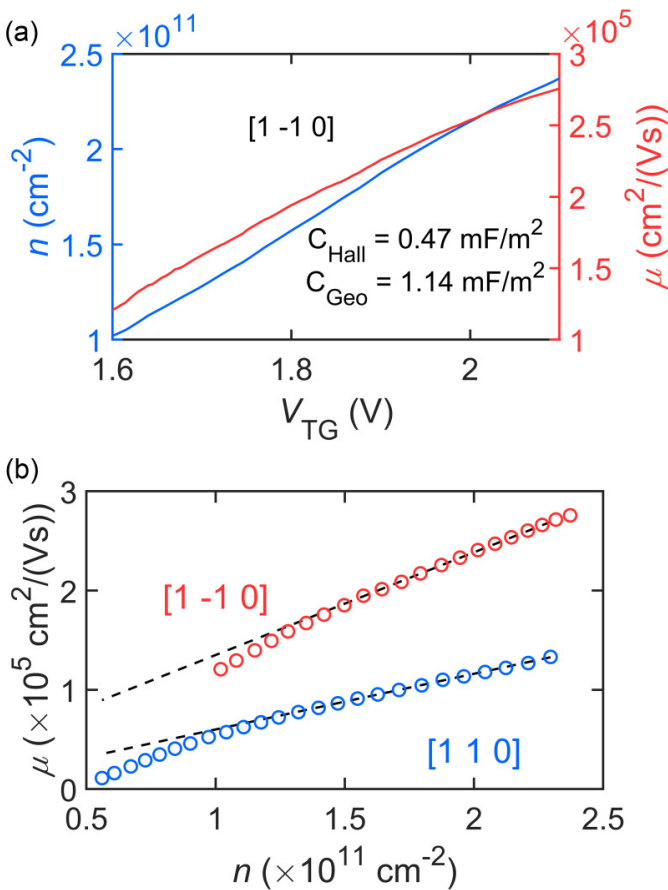

FIG. 1. (a) Data measured from the Hall bar along the $\left[\begin{array}{lll}1 & -1 & 0\end{array}\right]$ direction. The blue line shows the density $n$ as a function of the top gate voltage $V_{\mathrm{TG}}$. The Drude mobility $\mu$ is presented vs $V_{\mathrm{TG}}$ with the red line. There is a mismatch between the capacitance obtained from Hall measurement $C_{\mathrm{Hall}}$ and the parallel board model $C_{\mathrm{Geo}}$. (b) The plots of $\mu-n$ when the Hall bar is along [ $\left[\begin{array}{lll}1 & -1 & 0\end{array}\right]$ (red) and [ $\left.\begin{array}{lll}1 & 1 & 0\end{array}\right]$ (blue), respectively. Black dashed lines are a linear fit of $\mu-n$ with high density.

and the corresponding $\mu$ increases to $260000 \mathrm{~cm}^{2} / \mathrm{Vs}$. The density $n$ here is limited because of the breakthrough of the ALO dielectric layer when a large electric field was applied. Compared with Si-doped InSb of our previous work [16], the mobility increases by $\sim 60 \%$ for equivalent density. This mobility is more than twice as high as in the undoped wafer used in the work by Kulesh et al. [19] at the same density, and it is comparable with the previous doped wafer in Ref. [2] where, nevertheless, the carrier density cannot easily be tuned. In our samples, the gate capacitance $C_{\text {Hall }}$ was estimated to be $0.47 \mathrm{mF} / \mathrm{m}^{2}$ from the linear part of the $n-V_{\mathrm{TG}}$ line, which is only $\sim 40 \%$ of the value calculated from the geometry of the layer structure through a parallel-plate capacitor model $C_{\mathrm{Geo}}$. We found this mismatch between expected and measured capacitance consistently for a number of samples and substrates, doped and undoped structures. This mismatch, which has been reported previously by both our group and another group independently $[16,19]$, might be caused by screening between the top gates and the 2DEGs. Figure 1(b) shows the $n-\mu$ traces of both the Hall bars along crystal orientations

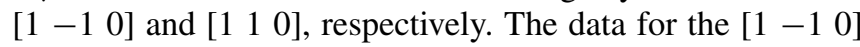
direction are the same as in Fig. 1(a). We find an anisotropy of the mobility in the two perpendicular crystal orientations, probably related to an anisotropic growth. For comparison, we also tried to grow undoped $\mathrm{InSb} \mathrm{QWs}$ on undoped $\mathrm{GaSb}$ substrates with the same growth sequence. Because there is much less lattice mismatch between $\mathrm{GaSb}$ and $\mathrm{InSb}$, the 
growth of the buffer layer is not necessary and therefore was not implemented. However, we did not manage to produce conducting Hall bars with the same microfabrication process. Interestingly, a 2DEG with mobility $\mu=330000 \mathrm{~cm}^{2} / \mathrm{Vs}$ at density $n=2.4 \times 10^{11} \mathrm{~cm}^{-2}$ is induced by $V_{\mathrm{TG}}$ in QWs grown on $\mathrm{GaSb}$ substrate when a tiny amount of modulation $\mathrm{Si}$ doping is added. Here, the doping dose is so low that a $2 \mathrm{DEG}$ cannot be induced when the top gate is grounded. However, the channel of the QW is switched on with a positive $V_{\mathrm{TG}}$ of $<1 \mathrm{~V}$ applied. The quality in terms of mobility of this InSb grown on GaSb substrate with tiny $\mathrm{Si}$ doping is even higher. Nevertheless, we find that the ohmic contacts on InSb QWs with GaSb substrate are much worse than those on GaAs substrates because the contacts do not work in magnetic fields $>3 \mathrm{~T}$.

\section{WAL MEASUREMENT}

The high-quality 2DEGs in undoped InSb QWs also display tunable SOI, which is characterized by WAL measurements. Data presented here are from the Hall bar along the [ $\left.\begin{array}{lll}1 & 1 & 0\end{array}\right]$ direction. Figure 2(a) presents the WAL effects at temperatures $T$ varying from 1.7 to $9 \mathrm{~K}$ for $n=2.1 \times 10^{11} \mathrm{~cm}^{-2}$. To increase the signal-to-noise ratio, each trace presented here is an average of $>5$ consecutive measurements. We find the conductivity peak of WAL at zero magnetic field by converting the longitudinal resistance and Hall resistance to longitudinal conductivity $\sigma_{x x}$ and subtracting a polynomial background. The height of the peak decreases with increasing $T$. The precise measurement of $\sigma_{x x}(B)$ enables us to extract coherence and SOI lengths using the Hikami-Larkin-Nagaoka (HLN) expression [22,23]. Similar methods are also adopted in papers where WAL effects are measured in doped InSb QWs [11] and InSb nanosheets [24]. The conductivity correction $\Delta \sigma_{x x}(B)$ of WALs reads

$$
\begin{aligned}
\Delta \sigma_{x x}(B)= & \frac{e^{2}}{2 \pi^{2} \hbar}\left[\psi\left(\frac{1}{2}+\frac{H_{\phi}}{B}+\frac{H_{\mathrm{SO}}}{B}\right)\right. \\
& +\frac{1}{2} \psi\left(\frac{1}{2}+\frac{H_{\phi}}{B}+\frac{2 H_{\mathrm{SO}}}{B}\right)-\frac{1}{2} \psi\left(\frac{1}{2}+\frac{H_{\phi}}{B}\right) \\
& -\ln \left(\frac{H_{\phi}+H_{\mathrm{SO}}}{B}\right)-\frac{1}{2} \ln \left(\frac{H_{\phi}+2 H_{\mathrm{SO}}}{B}\right) \\
& \left.+\frac{1}{2} \ln \left(\frac{H_{\phi}}{B}\right)\right] .
\end{aligned}
$$

Here, $H_{\phi}$ and $H_{\text {So }}$ are phase coherence field and spin-orbit field, respectively, and $\psi$ is the digamma function. These two fitting parameters can be converted to phase coherence length $l_{\phi}$ and spin-orbit length $l_{\text {SO }}$. Using $l_{\phi}=\sqrt{\frac{\hbar}{4 e H_{\phi}}}$, we present $l_{\phi}$ as a function of $T$ in Fig. 2(b) when $n=1.7 \times 10^{11}$ and $2.1 \times 10^{11} \mathrm{~cm}^{-2}$. The dashed line here is a fitting of the power law $l_{\phi} \propto T^{-\alpha}$. In both sets of measured data, $\alpha$ is very close to 1 . This implies that our system is in the standard 2D diffusive regime. A similar $l_{\phi} \propto 1 / T^{\alpha}$ relation is observed in other 2DEG systems [25-27]. Additionally, a three-parameter model by Iordanskii et al. [23], which in principle allows extracting Rashba and Dresselhaus contributions separately, does not give additional information for the range of data considered here.
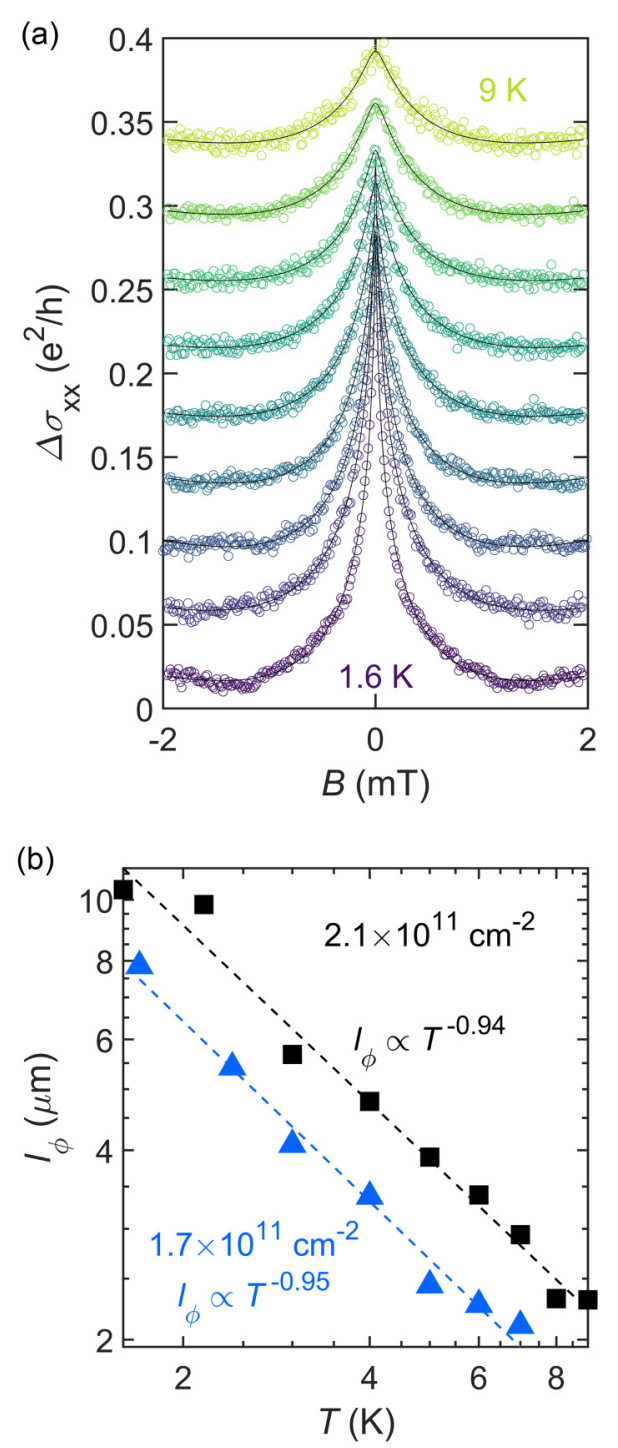

FIG. 2. (a) The temperature dependence of weak antilocalization with $n=2.1 \times 10^{11} \mathrm{~cm}^{-2}$. The open circles are data points, and the solid black lines are fits using the Hikami-Larkin-Nagaoka (HLN) expression. The traces have a constant offset for a better presentation. (b) Phase coherence length $l_{\phi}$ vs $T$ when $n=1.7 \times 10^{11} \mathrm{~cm}^{-2}$ (blue) and $2.1 \times 10^{11} \mathrm{~cm}^{-2}$ (black), respectively. The dashed line is the fitting with the power law $l_{\phi} \propto T^{-\alpha}$. The fitting shows that $\alpha=0.95$ and 0.94 when $n$ are $1.7 \times 10^{11}$ and $2.1 \times 10^{11} \mathrm{~cm}^{-2}$, respectively.

The SOI of the InSb 2DEG can be tuned by $n$ with $V_{\mathrm{TG}}$, which is probed with WAL measurements as well. Figure 3(a) shows the WAL with $n$ varying from $1.67 \times 10^{11}$ to $2.2 \times$ $10^{11} \mathrm{~cm}^{-2}$ at $1.7 \mathrm{~K}$. Here, we utilize the HLN expression again to analyze the SOI as a function of $n$. Figure 3(b) presents the splitting $\Delta_{\text {SO }}$ vs $n$ at the Fermi level due to SOI. Here, $\Delta_{\text {SO }}$ is obtained with $\Delta_{\mathrm{SO}}=\sqrt{\frac{2 \hbar^{2}}{\tau_{D} \tau_{\mathrm{SO}}}}$. Note that the spin-orbit time $\tau_{\mathrm{SO}}$ is calculated with the formula $\tau_{\mathrm{SO}}=\frac{\hbar}{4 e D H_{\mathrm{SO}}}$, where $D$ is the diffusion constant in 2D. With the increase of $n$ in the range of the measurements, $\Delta_{\text {SO }}$ increases from 0.5 to $0.74 \mathrm{meV}$. The values obtained here agree with the previous publication by Ke et al. [11], where their $\Delta_{\text {SO }}$ is slightly larger due to a higher $n$. Then we normalize $\Delta_{\text {SO }}$ to get the coefficient $\alpha_{\text {SO }}$ of 


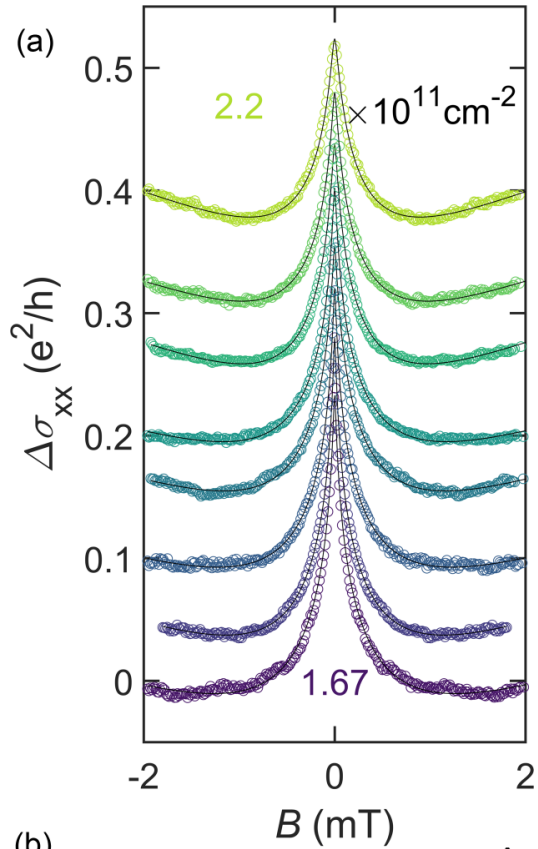

(b)

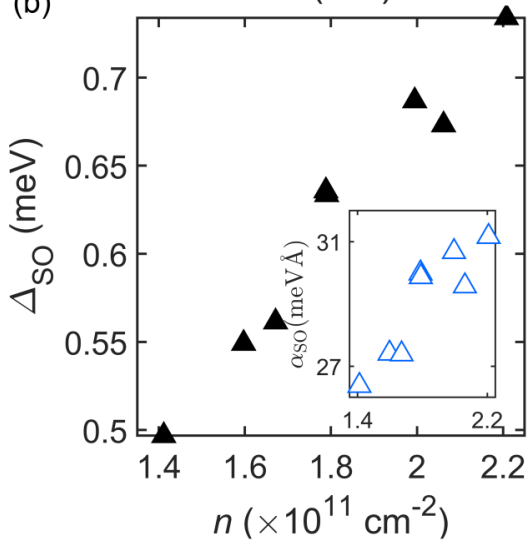

(c)

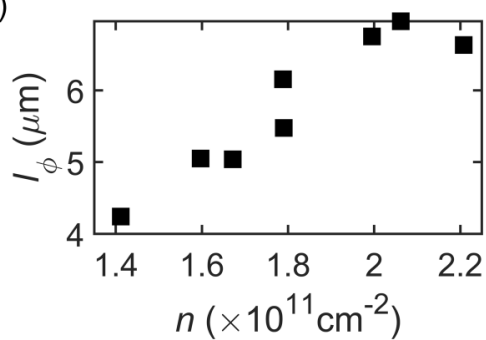

FIG. 3. (a) The density dependence of weak antilocalization with $T=1.7 \mathrm{~K}$. The open circles are data points. Black solid lines are fits using the Hikami-Larkin-Nagaoka (HLN) expression. The traces are vertically offset for clarity. (b) The spin-orbit splitting at the Fermi level $\Delta_{\text {So }}$ vs density $n$. A tendency that $\Delta_{\text {SO }}$ increases with the increase of $n$ is observed. Inset: the spin-orbit coefficient $\alpha_{\text {SO }}$ as a function of $n$. $\alpha_{\text {SO }}$ also grows with $n$. (c) Phase coherence length $l_{\phi}$ vs $n . l_{\phi}$ increases with higher $n$.

the SOI with $\alpha_{\mathrm{SO}}=\frac{\Delta_{\mathrm{SO}}}{2 k_{F}}$, where $k_{F}$ is the Fermi wave number. As shown in the inset of Fig. 3(b), $\alpha_{\mathrm{SO}}$ also increases when $n$ is higher. Because InSb is a material with both strong Rashba and Dresselhaus SOIs, we expect that the contribution of Rashba and Dresselhaus SOIs are comparable. Additionally, since the Dresselhaus coefficient $\beta_{D}$ is an intrinsic material parameter, the increase of $\alpha_{\mathrm{SO}}$ is likely due to a larger Rashba contribution from a higher electric field in the high-density regime. Furthermore, as presented in Fig. 3(c), $l_{\phi}$ increases when $n$ is higher, which means that the decoherence is reduced with higher density and mobility.

\section{SCATTERING MECHANISM ANALYSIS}

Next, we study these high-quality InSb 2DEGs with magnetotransport measurements in magnetic fields up to $8 \mathrm{~T}$. Here, we present measurements on the Hall bar along the $\left[\begin{array}{lll}1 & -1 & 0\end{array}\right]$ direction. As shown in Fig. 1(b) before, in our 2DEGs, $\mu$ is proportional to $n$. This indicates that long-range remote ionized impurity scattering and short-range background impurity scattering are comparable [28-30]. More insight into the scattering mechanisms can be obtained from analyzing $\mathrm{SdH}$ oscillations with the Ando formula [27]. Figure 4(a) presents the effective mass of InSb 2DEGs $m^{*}$ as a function of magnetic field $B$ when $n$ is $1.4 \times 10^{11}$ and $2.1 \times 10^{11} \mathrm{~cm}^{-2}$. The method to measure $m^{*}$ is like our previous publications [16,31]. In the range with small amplitude $\mathrm{SdH}$ oscillations compared with the magnetoresistance background, $m^{*}$ has a roughly constant value of $0.015 m_{e}$ in both low- and high-density regimes, where $m_{e}$ is the free electron mass. This shows that the nonparabolicity of the conduction band is still negligible within the range of $n$ in which we are interested. This value agrees with the $\mathbf{k} \cdot \mathbf{p}$ calculations and our previous measurement in QWs with the same width [16]. With the obtained $m^{*}$ plugged into the Ando formula, the quantum lifetime of Landau levels $\tau_{q}$ is extracted in the following. Figure 4(b) shows $\left|\Delta \rho_{x x}\right| /\left[\bar{\rho}_{x x} f(B, T)\right]$ vs $1 / B$ with $n=1.4 \times 10^{11} \mathrm{~cm}^{-2}$ at $2.1 \mathrm{~K}$. Here, $\Delta \rho_{x x}$ is the oscillating part of the magnetoresistance obtained by subtracting a polynomial background $\bar{\rho}_{x x}$, and

$$
f(B, T)=\frac{\frac{2 \pi^{2} k_{B} T}{\hbar \omega_{c}}}{\sinh \left(\frac{2 \pi^{2} k_{B} T}{\hbar \omega_{c}}\right)} .
$$

Then $\tau_{q}$ is extracted to be $0.067 \mathrm{ps}$ using a linear fit. This value is much smaller than the Drude mean free time $\tau_{D}$, which is defined as the mean time interval between two scattering events. Here, $\tau_{D}$ is calculated to be $1.26 \mathrm{ps}$ from the mobility $\mu$ with the same $n$ using the formula $\tau_{D}=m^{*} \mu / e$. The large ratio of $\tau_{D}$ vs $\tau_{q}$ implies a significant contribution of longrange scattering $[32,33]$.

Knowing $\tau_{q}$, we can estimate the $g$ factor of the 2DEGs from an activation energy measurement. Figure 4(c) plots the longitudinal conductance $\sigma_{x x}$ of filling factor $v=1$ and 2 as a function of $T$ with $n=1.4 \times 10^{11} \mathrm{~cm}^{-2}$ he $\sigma_{x x}-T$ diagrams follow the expression $\sigma_{x x} \propto \exp \left[-E_{A, 1(2)} / 2 k_{B} T\right]$, where $E_{A, 1(2)}$ is the activation energy of filling factor $v=1(2)$. From the linear fit in Fig. 4(c), $E_{A, 1}$ and $E_{A, 2}$ are found to be 12.78 and $8.41 \mathrm{meV}$, respectively. Without considering the Landau level broadening due to scattering, we can write $g^{*} \mu_{B} B \approx E_{A, 1}$ at filling factor $v=1$. Therefore, the $g$ factor at filling factor $v=1$ is $g^{*} \sim 36$. Though this is a lower limit of $g^{*}$, it is already close to the value calculated from the $\mathbf{k} \cdot \mathbf{p}$ model [16]. A better estimation of the $g$ factor through the activation energy is the following. As shown in Fig. 4(d) schematically, with the consideration of a Landau level broadening $\Gamma$, the activation energies $E_{\mathrm{A}, 1}$ and $E_{\mathrm{A}, 2}$ 
(a)
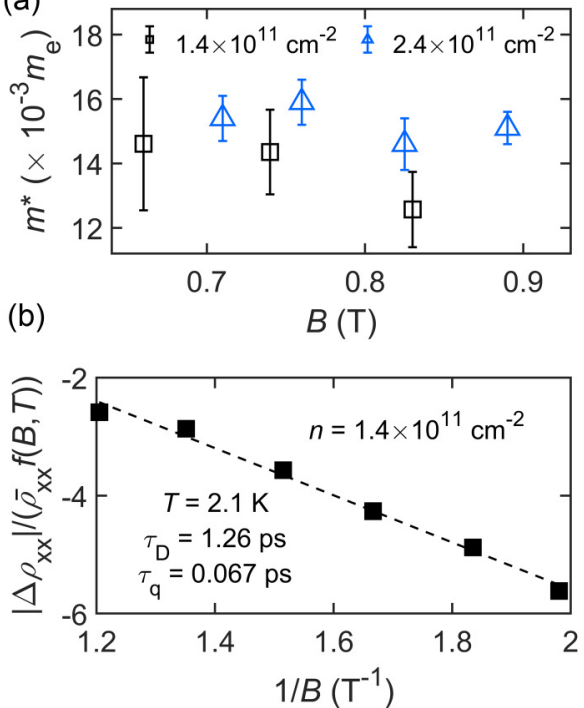

(c)

(d)

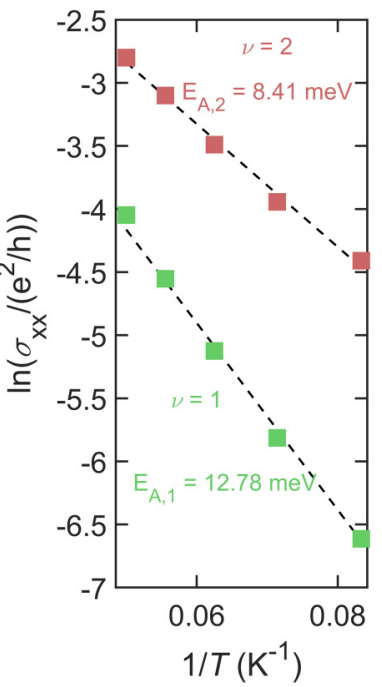

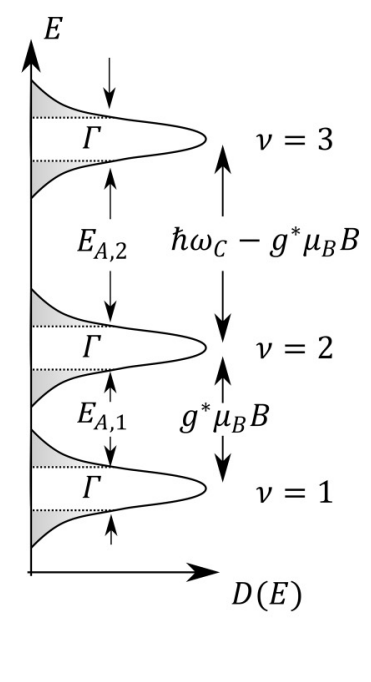

FIG. 4. (a) Effective mass $m^{*}$ obtained from Ando formula fitting as a function of the magnetic field $B$. Two sets of data with $n=1.4 \times$ $10^{11} \mathrm{~cm}^{-2}$ (black) and $2.4 \times 10^{11} \mathrm{~cm}^{-2}$ (blue) are measured. Within the error bar from fitting, we determine $m^{*}=0.015 m_{e}$ as a constant value within the measurement range of our experiment. (b) The fitting to obtain quantum lifetime $\tau_{q}$ with $n=1.4 \times 10^{11} \mathrm{~cm}^{-2}$. The method is introduced in the main text. Here, the black squares are the data points, and the dashed line is a linear fitting. (c) Temperature dependences of the longitudinal conductivity $\sigma_{x x}$ when $v=2$ (red) and $v=1$ (green), respectively. The activation energies $E_{A, 2(1)}$ are obtained from the linear fits (dashed lines) presented in the figure. (d) A schematic diagram to depict the relationship between the Landau level broadening $\Gamma$, Zeeman energy $g^{*} \mu_{B} B$, Landau energy $\hbar \omega_{c}$, and the measured activation energy $E_{\mathrm{A}, 2}$ and $E_{\mathrm{A}, 1}$. In the figure, $E$ is the energy, and $D(E)$ is the density of states.

then $\operatorname{read} E_{A, 1}=g^{*} \mu_{B} B-\Gamma$ and $E_{A, 2}=\hbar \omega_{c}-g^{*} \mu_{B} B-\Gamma$, respectively. Here, $\hbar \omega_{c}$ is the Landau level energy. Assuming $\Gamma=\sqrt{\frac{1}{2 \pi} \hbar \omega_{c} \frac{\hbar}{\tau_{q}}}$ as suggested in Ref. [27], $g^{*}$ for $v=1$ and 2 are calculated to be $\sim 64$ and 46 , respectively. These values are larger than the calculation from the single-particle picture, where $g^{*} \sim 39$ with the same width of QWs. More about the larger $g$ factor will be discussed in the following section.

\section{COINCIDENCE MEASUREMENT}

Next, we present our results of the coincidence measurement on the Hall bar along the $[1-10]$ direction. This method has been introduced in previous publications [16,34-36]. The Landau level energy is proportional to the perpendicular magnetic field $B_{\perp}$, while the Zeeman energy is proportional to the total magnetic field $B_{\text {tot }}$. The ratio between these two energies can be changed continuously by changing the angle $\theta$ between the direction of sample normal and the total magnetic field $B_{\text {tot }}$ [inset of Fig. 5(a)]. Therefore, $B_{\perp}$, the projection of the magnetic field along the sample normal, is $B_{\perp}=B_{\text {tot }} \cos (\theta)$. Here, we introduce a parameter $r=g^{*} \mu_{B} B_{\text {tot }} / \hbar \omega_{c}$. Hence, we obtain $r \cos (\theta)=g^{*} m^{*} / 2 m_{e}$. The number of $r$ can be obtained by monitoring the behavior of local maxima and minima of $\rho_{x x}$ at different tilt angles $\theta$. In a single-particle picture, for instance, at $r=1$, i.e., when the Zeeman energy equals the Landau energy, the minima of $\mathrm{SdH}$ traces of longitudinal resistivity $\rho_{x x}$ will only occur at odd filling factors, such as $v=3,5$, and 7 . In contrast, at $r=2$, the minima of $\rho_{x x}$ occur at even filling factors, such as $v=4,6$, and 8. Additionally, the minima of $\rho_{x x}$ for $v=1$ and 2 remain the minima when $r=2$ because the gaps are always open.
Figure 5(a) depicts the dependence of $\rho_{x x}$ on $B_{\perp}$ with a continuous change of $\theta$ at $0.5 \mathrm{~K}$ when $n=2.4 \times 10^{11} \mathrm{~cm}^{-2}$. The value of $\theta$ is calibrated with the slope of the Hall trace at low magnetic field with high accuracy. For a better determination of $r=1$ and 2, we extract the $\rho_{x x}$ with even $(v=4$,

(a)
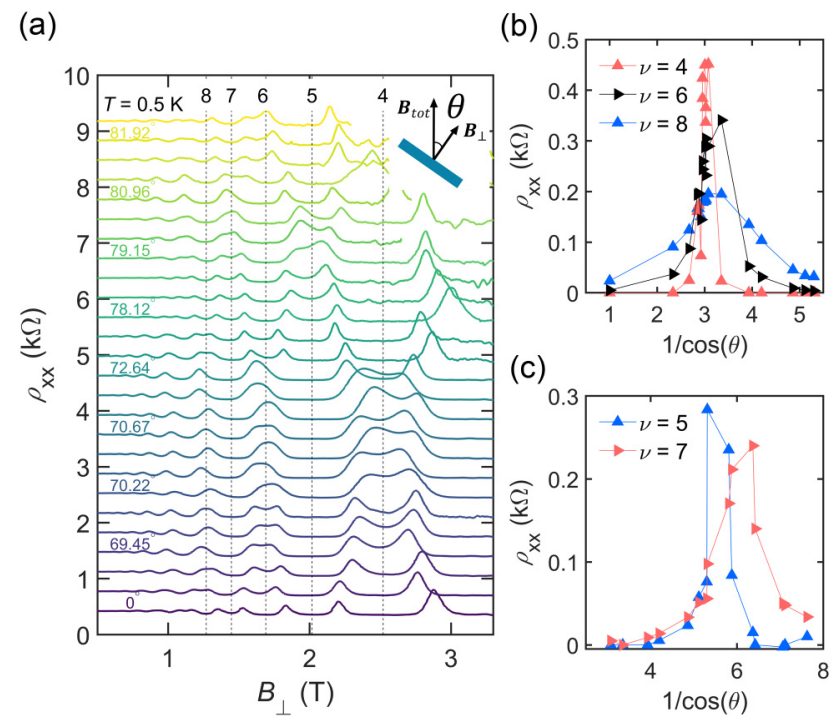

(c)

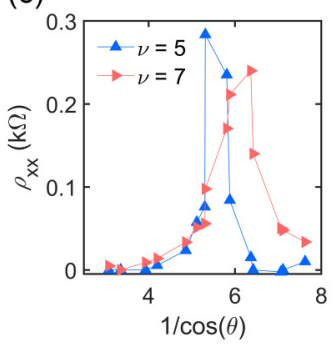

FIG. 5. (a) The Shubnikov-de Haas ( $\mathrm{SdH})$ oscillations measured for different tilt angles. The traces have a constant offset of $350 \Omega$ for a better presentation. The definition of the tilted angle $\theta$ is presented in the inset. (b) The longitudinal resistance $\rho_{x x}$ with even filling factors $v=4,6$, and 8 vs the tilted angle $\theta$. (b) The longitudinal resistance $\rho_{x x}$ with odd filling factors $v=5$ and 7 vs the tilted angle $\theta$. 


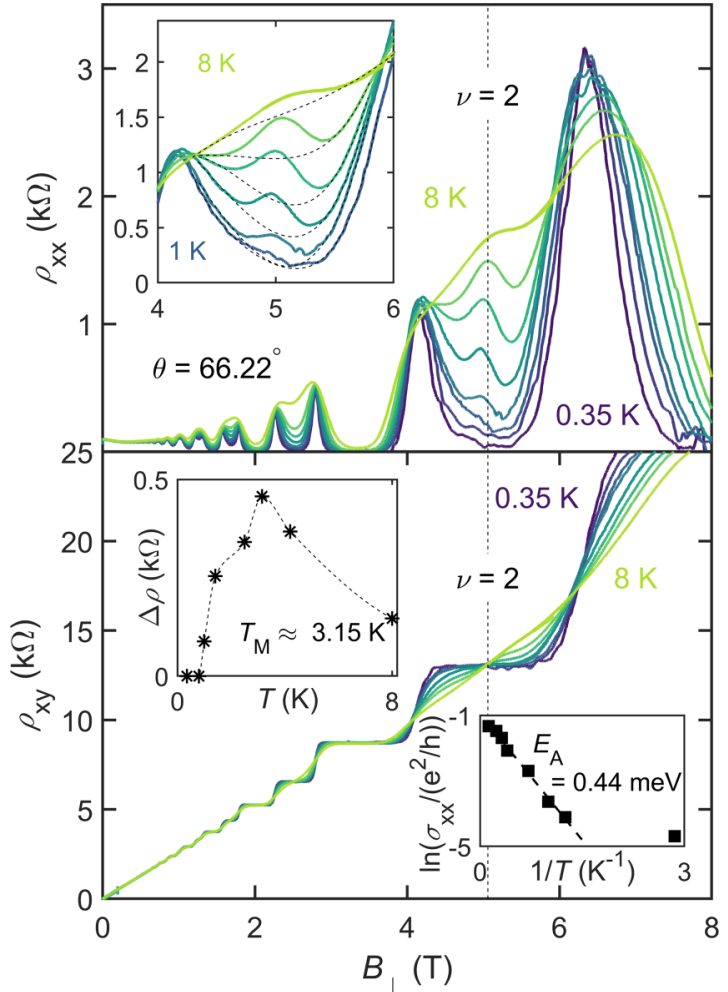

FIG. 6. The temperature dependence of the longitudinal and transversal resistivity $\rho_{x x}$ and $\rho_{x y}$ when $\theta=66.22^{\circ}$. Here, the coincidence $r=1$ for $v=2$ is achieved. Inset of the upper panel: the zoom-in of $\rho_{x x}-B_{\perp}$ diagram around $v=2$ with the same axis. The black dashed line is a polynomial background to show the resistivity peak. Upper inset of the lower panel: the peak height $\Delta \rho$ as a function of $T$. The Curie temperature $T_{\mathrm{C}}$ is determined to be $T_{C} \sim 3.15 \mathrm{~K}$, where $\Delta \rho$ achieves its maximum. Here, the stars are data, and the dashed line is guidance to eyes. Lower inset of the lower panel: analysis of $\sigma_{x x}$ vs $T$ with the gap model when $v=2$. The squares are the data, and the dashed line is a linear fitting.

6 , and 8$)$ and odd filling factors ( $v=5$ and 7) and plot them in Figs. 5(b) and 5(c) as functions of $\theta$. For each even filling factor, the coincidence of $r=1$ is found by picking the $\theta$ at which $\rho_{x x}$ reaches its maximum. Similarly, the coincidence of $r=2$ of each odd filling factor is found where $\rho_{x x}$ has a maximum. The error bar of each coincidence angle is determined by the step of $\theta$ between the coincidence and the nearest data points around it.

Moreover, the gap at $v=2$ stays open at low temperature with the coincidence condition $r=1$. This is different from the situations of $v=4,6$, and 8 . For $v=2, \rho_{x x}$ stays zero for all tilt angles. Nevertheless, the minimum of $\rho_{x x}$ at $v=2$ starts to increase above zero when $T>1 \mathrm{~K}$. Through reproducing the $\mathrm{SdH}$ measurements at 1.5 and $4.2 \mathrm{~K}$ with a fine-tuning of $\theta$, we determine that the gap at $v=2$ reaches its minimum when $\theta=66.22^{\circ}$, where the uncertainty of $\theta$ is $<0.5^{\circ}$. This also means that the coincidence condition of $r=1$ is most precisely achieved at this angle. Figure 6 depicts the $T$ dependence of the longitudinal resistivity $\rho_{x x}$ and transversal resistivity $\rho_{x y}$ with the coincidence condition $r=1$ for filling factor $v=2$. At filling factor $v=2, \rho_{x x}$ increases with increasing $T$ from 0.35 to $8 \mathrm{~K}$. Meanwhile, the Hall plateau at $v=2$ becomes narrower and approaches the classical Hall effect at $8 \mathrm{~K}$, though plateaus for higher filling factors, such as $v=3$ and 5 , are still pronounced. This is different from the previous paper by Chokomakoua et al. [37], where an InSb QW in van der Pauw geometry was studied. There, the Hall plateau of $v=2$ was not well defined at low temperatures. However, our results are like the work by Koch et al. [38], where the Landau-level anticrossing due to a magnetic instability is found in GaInAs/InP heterostructures.

In addition to a nonvanishing gap at filling factor $v=2$ with $r=1$, a resistivity peak appears at intermediate temperature but vanishes at high temperature in $\rho_{x x}-B_{\perp}$ traces. This agrees with the phenomenon of quantum Hall Ising ferromagnetism $[39,40]$. The inset in the upper panel of Fig. 6 depicts this process. The appearance and vanishing of the resistivity spikes can be quantitatively depicted using Curie temperature $T_{\mathrm{C}}$. Here, we adopt the methods introduced by De Poortere et al. [40] to estimate $T_{\mathrm{C}}$. The heights $\Delta \rho$ of the resistivity peaks at different temperatures are obtained by subtracting a polynomial magnetoresistance background around $v=2$ [black dashed line in the inset of the upper panel of Fig. 6(a)] from the measured $\rho_{x x}$. Plots of $\Delta \rho$ vs $T$ are shown in the upper inset of the lower panel of Fig. 6 . When $T=T_{\mathrm{M}}=3.15 \mathrm{~K}$, $\Delta \rho$ reaches its maximum. Meanwhile, the width of the resistivity spike starts to increase when $T \geqslant T_{M}=3.15 \mathrm{~K}$ as well. Therefore, we estimate the Curie temperature $T_{\mathrm{C}}$ to be around a value $T_{\mathrm{C}} \cong T_{M} \sim 3 \mathrm{~K}$. Similar phenomena are observed in

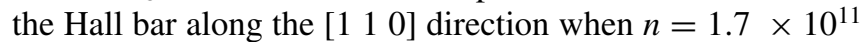
and $2.2 \times 10^{11} \mathrm{~cm}^{-2}$, respectively. Though the phenomenon of Ising ferromagnetism is less pronounced at lower density, we still managed to estimate $T_{\mathrm{C}}$ to be $\sim 3 \mathrm{~K}$ when $n=1.7 \times$ $10^{11} \mathrm{~cm}^{-2}$. In addition, the $T$ dependence of $\rho_{x x}$ at $v=2$ meets the thermal activation [41] model numerically within the accuracy of our measurement. Like Fig. 4(c), we analyze the $\sigma_{x x}-T$ diagram with $\sigma_{x x} \propto \exp \left(-E_{A} / 2 k_{B} T\right)$ and obtain $E_{A}=$ $0.44 \mathrm{meV}$ (lower inset of the lower panel in Fig. 6). We reproduced this measurement when $n=1.7 \times 10^{11} \mathrm{~cm}^{-2}$, finding that $E_{\mathrm{A}}=0.18 \mathrm{meV}$.

With the coincidence condition of $r=1$ at $v=2$ included, we investigate the spin-polarization dependence of the spin susceptibility $\chi$. From the data presented in Fig. 5, the coincidence angles with different $r$ and $v$ are extracted. Using $r \cos (\theta)=g^{*} m^{*} / 2 m_{e}, g^{*} m^{*}$ is obtained. Because $\chi=\frac{g^{*} m^{*}}{2 \pi^{2}}$ in our 2D system, we use $g^{*} m^{*}$ to describe the spin susceptibility in this paper. Figure 7(a) shows the traces of $g^{*} m^{*}$ as a function of spin polarization $P$ when $n$ is $2.4 \times 10^{11} \mathrm{~cm}^{-2}$ (blue) and $1.4 \times 10^{11} \mathrm{~cm}^{-2}$ (red). Here, data points are obtained from two same Hall bars along the $\left[\begin{array}{lll}1 & -1 & 0\end{array}\right]$ direction. As introduced by Zhu et al. [42], $P$ can be calculated easily through $P=r / \nu$. In both traces presented, $g^{*} m^{*}$ grows with $P$. For a better illustration, we present $g^{*}$ along the right axis, assuming that $m^{*}$, measured to be $0.015 m_{e}$ from SdH oscillations, is also independent of $P$. For low magnetic fields, $g^{*}$ is $\sim 40$ in both cases, which agrees with the calculation within a k $\cdot$ p model. Nevertheless, when $P=0.5, g^{*}$ increases significantly up to $\sim 53$ and $\sim 46$ with $n=2.4 \times 10^{11}$ and $1.4 \times$ $10^{11} \mathrm{~cm}^{-2}$, respectively. This $g^{*}-n$ relation agrees with the publications by Yang et al. [36] and Nedniyom et al. [43]. 
(a)

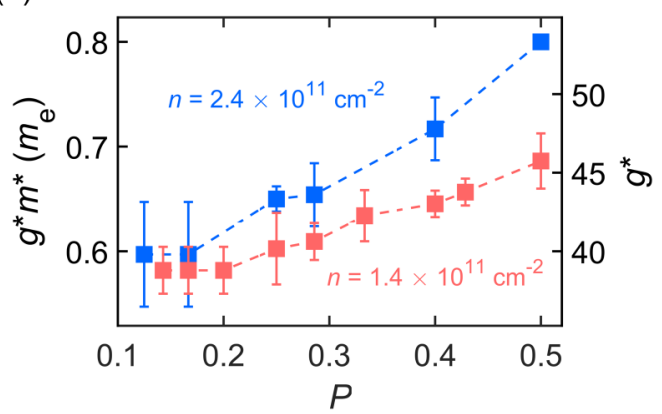

(b)

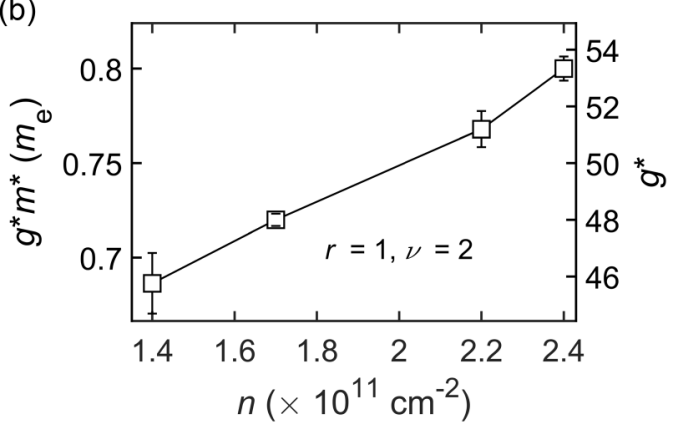

FIG. 7. (a) $g^{*} m^{*}$ obtained from coincidence measurement vs the spin polarization $P$ when $n=2.4 \times 10^{11} \mathrm{~cm}^{-2}$ (blue) and $1.4 \times$ $10^{11} \mathrm{~cm}^{-2}$ (red), respectively. Plugging in $m^{*}=0.015 m_{e}, g^{*}$ is obtained and shown in the axis of the right-hand side. (b) $g^{*} m^{*}$ and $g^{*}$ vs $n$. Here, we present the coincidence where $r=1$ and $v=2 . m^{*}$ is still assumed to be $0.015 m_{e}$.

Furthermore, the spin susceptibility also grows with $n$. We summarize all the $g^{*} m^{*}$ measured with $r=1$ and $v=2$ from three samples with the same quality and plot them as a function of $n$ in Fig. 7(b). Assuming that $m^{*}$ is constant at $0.015 m_{e}, g^{*}$ is calculated and plotted too. With the increase of $n$ from $1.4 \times 10^{11}$ to $2.4 \times 10^{11} \mathrm{~cm}^{-2}, g^{*} m^{*}$ increases by $\sim 15 \%$. This tendency is opposite to the results from dilute 2DEGs in GaAs, where $g^{*} m^{*}$ decreases with the increase of $n$ [42]. In GaAs 2DEGs, the interaction parameter $r_{\mathrm{s}}$, defined as the ratio between Coulomb energy and Fermi energy, is smaller when $n$ is higher. Nevertheless, due to the light effective mass and the high density in InSb 2DEGs, $r_{\mathrm{s}}$ is only between 0.17 and 0.23 in our experiment. This value is not only much smaller than those of low-density 2DEG in GaAs and AlAs, but the variation is also limited in our experiment. Therefore, the contribution of electron-electron interaction to the $n$ dependence of the spin susceptibility is not dominant. Instead of electron-electron interactions, this $g^{*} m^{*}-n$ diagram is more likely to be influenced by the SOI. As we verified through WAL measurement before, the SOI is stronger with increasing $n$. Effectively, this extra contribution is probed as an enlarged Zeeman energy through the coincidence measurement, leading to a larger $g$ factor. Similar phenomena have been observed in previous work in an InAs 2DEG [44], another narrow bandgap material with light effective mass and strong SOI, where the anticrossing of Landau levels in titled fields was also found.

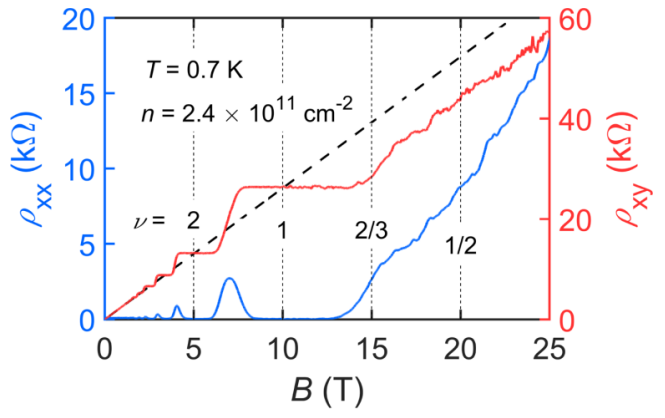

FIG. 8. $\rho_{x x}$ and $\rho_{x y}$ measurement of the InSb quantum well (QW) in a large magnetic field range at the temperatures of $0.7 \mathrm{~K}$ with $n=2.4 \times 10^{11} \mathrm{~cm}^{-2}$. The dashed line is an extrapolation of the Hall trace in the small magnetic field.

\section{MAGNETOTRANSPORT WHEN $v<1$}

Finally, motivated by the high mobility of our 2DEGs, we probe the behavior of the 2DEGs in even higher perpendicular magnetic fields. Figure 8 depicts one of the magnetoresistance measurements of our Hall bar along the $\left[\begin{array}{lll}1 & -1 & 0\end{array}\right]$ direction at $0.7 \mathrm{~K}$ with $n=2.4 \times 10^{11} \mathrm{~cm}^{-2}$. In $B$ fields where $v<1$, the ohmic contacts are still working properly, indicating the metallic behavior of the sample [45]. However, despite the high quality of the sample, there are still no signatures related to FQHEs. The Hall resistance $\rho_{x y}$ increases with increasing $B$ when $v<1$. However, it is only approaching but not reaching the classical limit (dashed black line). Meanwhile, there is no convincing local minimum in $\rho_{x x}$ or plateau in $\rho_{x y}$ when $B>15 \mathrm{~T}$. The absence of any FQHE-related features shows that there is still room to improve the sample quality. We notice that the weak electron-electron interaction in $\mathrm{InSb}$ may not be advantageous to form fractional states. Furthermore, the quantum lifetime of InSb QWs is still not as long compared with systems where the FQHE has been observed, such as the pioneering works in $\mathrm{GaAs}, \mathrm{Si} / \mathrm{Ge}$, and graphene [46-48]. The wide integer quantum Hall plateaus also indicate disorder hampering the formation of FQHEs in our devices. More optimized growth techniques are required to increase the chance in $\mathrm{InSb}$ to explore fractional states.

\section{CONCLUSIONS}

In conclusion, we have presented transport experiments on gate-tunable high-quality 2DEGs in undoped InSb QWs. With the elimination of Si modulation doping, the mobility is significantly increased. Tunable SOIs were probed through WAL measurements in a 2D diffusive transport regime. Furthermore, using coincidence methods, we find that the $g$ factor grows with both the spin polarization and carrier density. For filling factor $v=2$, a signature of Ising quantum Hall ferromagnetic phase was observed in a tilted magnetic field, and the Curie temperature was estimated. Finally, despite the high mobility, the undoped InSb QWs still did not exhibit fractional quantum Hall states up to magnetic fields of $25 \mathrm{~T}$.

\section{ACKNOWLEDGMENTS}

We thank Dr. F. K. de Vries, Dr. C. Reichl, and Mr. L. Ginzburg for fruitful discussions. We appreciate Mr. P. 
Märki and Mr. T. Bähler for their technical supports. We acknowledge the support of HFML-RU/NWO-I, member of the European Magnetic Field Laboratory. We acknowledge fund- ing from QuantERA. This paper was supported by the Swiss National Science Foundation through the National Center of Competence in Research Quantum Science and Technology.
[1] O. Madelung, U. Roessler, and M. Schulz, Landolt-BernsteinGroup III Condensed Matter (Springer, Berlin, 2000), 41D.

[2] A. M. Gilbertson, W. R. Branford, M. Fearn, L. Buckle, P. D. Buckle, T. Ashley, and L. F. Cohen, Zero-field spin splitting and spin-dependent broadening in high-mobility $\mathrm{InSb} / \mathrm{In}_{1-x} \mathrm{Al}_{x} \mathrm{Sb}$ asymmetric quantum well heterostructures, Phys. Rev. B 79, 235333 (2009).

[3] M. Leontiadou, K. Litvinenko, A. Gilbertson, C. Pidgeon, W. Branford, L. Cohen, M. Fearn, T. Ashley, M. Emeny, B. Murdin et al., Experimental determination of the Rashba coefficient in InSb/InAlSb quantum wells at zero magnetic field and elevated temperatures, J. Phys.: Condens. Matter 23, 035801 (2011).

[4] G. A. Khodaparast, R. E. Doezema, S. J. Chung, K. J. Goldammer, and M. B. Santos, Spectroscopy of Rashba spin splitting in InSb quantum wells, Phys. Rev. B 70, 155322 (2004).

[5] R. L. Kallaher, J. J. Heremans, N. Goel, S. J. Chung, and M. B. Santos, Spin-orbit interaction determined by antilocalization in an InSb quantum well, Phys. Rev. B 81, 075303 (2010).

[6] H. Chen, J. Heremans, J. Peters, A. Govorov, N. Goel, S. Chung, and M. Santos, Spin-polarized reflection in a twodimensional electron system, Appl. Phys. Lett. 86, 032113 (2005).

[7] I. Zutic, J. Fabian, and S. D. Sarma, Spintronics: Fundamentals and applications, Rev. Mod. Phys. 76, 323 (2004).

[8] R. Lutchyn, E. P. A. M. Bakkers, L. P. Kouwenhoven, P. Krogstrup, C. M. Marcus, and Y. Oreg, Majorana zero modes in superconductor-semiconductor heterostructures, Nat. Rev. Mater. 3, 52 (2018).

[9] Y. Oreg, G. Refael, and F. von Oppen, Helical Liquids and Majorana Bound States in Quantum Wires, Phys. Rev. Lett. 105, 177002 (2010).

[10] M. Deng, S. Vaitiekenas, E. B. Hansen, J. Danon, M. Leijnse, K. Flensberg, J. Nygård, P. Krogstrup, and C. M. Marcus, Majorana bound state in a coupled quantum-dot hybrid-nanowire system, Science 354, 1557 (2016).

[11] C. T. Ke, C. M. Moehle, F. K. de Vries, C. Thomas, S. Metti, C. R. Guinn, R. Kallaher, M. Lodari, G. Scappucci, T. Wang et al., Ballistic superconductivity and tunable $\pi$-junctions in InSb quantum wells, Nat. Commun. 10, 3764 (2019).

[12] A. Fornieri, A. M. Whiticar, F. Setiawan, E. Portolés, A. C. C. Drachmann, A. Keselman, S. Gronin, C. Thomas, T. Wang, R. Kallaher, G. C. Gardner, E. Berg, M. J. Manfra, A. Stern, C. M. Marcus, and F. Nichele, Evidence of topological superconductivity in planar Josephson junctions, Nature 569, 89 (2019).

[13] C. A. Lehner, T. Tschirky, T. Ihn, W. Dietsche, J. Keller, S. Fält, and W. Wegscheider, Limiting scattering processes in highmobility InSb quantum wells grown on GaSb buffer systems, Phys. Rev. Materials 2, 054601 (2018).

[14] T. Tschirky, S. Mueller, Ch. A. Lehner, S. Falt, T. Ihn, K. Ensslin, and W. Wegscheider, Scattering mechanisms of highest-mobility InAs $/ \mathrm{Al}_{x} \mathrm{Ga}_{1-x} \mathrm{Sb}$ quantum wells, Phys. Rev. B 95, 115304 (2017).
[15] C. Thomas, A. T. Hatke, A. Tuaz, R. Kallaher, T. Wu, T. Wang, R. E. Diaz, C. G. Gardner, M. A. Capano, and M. J. Manfra, High-mobility InAs 2DEGs on GaSb substrates: A platform for mesoscopic quantum transport, Phys. Rev. Materials 2, 104602 (2018).

[16] Z. Lei, C. A. Lehner, K. Rubi, E. Cheah, M. Karalic, C. Mittag, L. Alt, J. Scharnetzky, P. Märki, U. Zeitler, W. Wegscheider, T. Ihn, and K. Ensslin, Electronic $g$ factor and magnetotransport in InSb quantum wells, Phys. Rev. Research 2, 033213 (2020).

[17] F. Qu, J. van Veen, F. K. de Vries, A. J. Beukman, M. Wimmer, W. Yi, A. A. Kiselev, B.-M. Nguyen, M. Sokolich, M. J. Manfra, F. Nichele, C. M. Marcus, and L. P. Kouwenhoven, Quantized conductance and large $g$-factor anisotropy in InSb quantum point contacts, Nano Lett. 16, 7509 (2016).

[18] Z. Lei, C. A. Lehner, E. Cheah, C. Mittag, M. Karalic, W. Wegscheider, K. Ensslin, and T. Ihn, Gate-defined quantum point contact in an InSb two-dimensional electron gas, Phys. Rev. Research 3, 023042 (2021).

[19] I. Kulesh, C. T. Ke, C. Thomas, S. Karwal, C. M. Moehle, S. Metti, R. Kallaher, G. C. Gardner, M. J. Manfra, and S. Goswami, Quantum Dots in an InSb Two-Dimensional Electron Gas, Phys. Rev. Appl. 13, 041003(R) (2020).

[20] S. Sarkozy, K. Das Gupta, C. Siegert, A. Ghosh, M. Pepper, I. Farrer, H. E. Beere, D. A. Ritchie, and G. A. C. Jones, Low temperature transport in undoped mesoscopic structures, Appl. Phys. Lett. 94, 172105 (2009).

[21] S. Peters, L. Tiemann, C. Reichl, S. Fält, W. Dietsche, and W. Wegscheider, Improvement of the transport properties of a high-mobility electron system by intentional parallel conduction, Appl. Phys. Lett. 110, 042106 (2017).

[22] S. Hikami, A. I. Larkin, and Y. Nagaoka, Spin-orbit interaction and magnetoresistance in the two dimensional random system, Prog. Theor. Phys. 63, 707 (1980).

[23] S. V. Iordanskii, Yu. B. Lyanda-Geller, and G. E. Pikus, Weak localization in quantum wells with spin-orbit interaction, Pis'ma Zh. Eksp. Teor. Fiz. 60, 199 (1994).

[24] Y. Chen, S. Huang, D. Pan, J. Xue, L. Zhang, J. Zhao, and H. Q. Xu, Strong and tunable spin-orbit interaction in a single crystalline InSb nanosheet, npj 2D Mater. Appl. 5, 3 (2021).

[25] V. Senz, T. Heinzel, T. Ihn, K. Ensslin, G. Dehlinger, D. Grützmacher, and U. Gennser, Coexistence of weak localization and a metallic phase in $\mathrm{Si} / \mathrm{SiGe}$ quantum wells, Phys. Rev. B 61, R5082(R) (2000).

[26] V. Senz, T. Ihn, T. Heinzel, K. Ensslin, G. Dehlinger, D. Grützmacher, and U. Gennser, Analysis of the Metallic Phase of Two-Dimensional Holes in SiGe in Terms of Temperature Dependent Screening, Phys. Rev. Lett. 85, 4357 (2000).

[27] T. Ihn, Semiconductor Nanostructures: Quantum States and Electronic Transport (Oxford University Press, Oxford, 2010). 
[28] E. H. Hwang and S. Das Sarma, Limit to two-dimensional mobility in modulation-doped GaAs quantum structures: How to achieve a mobility of 100 million, Phys. Rev. B 77, 235437 (2008).

[29] L. Pfeiffer, K. W. West, H. L. Stormer, and K. W. Baldwin, Electron mobilities exceeding $10^{7} \mathrm{~cm}^{2} / \mathrm{V} \mathrm{s}$ in modulationdoped GaAs, Appl. Phys. Lett. 55, 1888 (1989).

[30] V. Umansky, R. de-Picciotto, and M. Heiblum, Extremely highmobility two dimensional electron gas: Evaluation of scattering mechanisms, Appl. Phys. Lett. 71, 683 (1997).

[31] Z. Lei, C. A. Lehner, E. Cheah, M. Karalic, C. Mittag, L. Alt, J. Scharnetzky, W. Wegscheider, T. Ihn, and K. Ensslin, Quantum transport in high-quality shallow InSb quantum wells, Appl. Phys. Lett. 115, 012101 (2019).

[32] P. T. Coleridge, Small-angle scattering in two-dimensional electron gases, Phys. Rev. B 44, 3793 (1991).

[33] S. Das Sarma and F. Stern, Single-particle relaxation time versus scattering time in an impure electron gas, Phys. Rev. B 32, 8442 (1985).

[34] H. W. Schumacher, A. Nauena, U. Zeitler, R. J. Haug, P. Weitz, A. G. M. Jansen, and F. Schäfflerd, Anomalous coincidences between valley split Landau levels in a $\mathrm{Si} / \mathrm{SiGe}$ heterostructure, Phys. B: Condens. Matter 256-258, 260 (1998).

[35] S. Brosig, K. Ensslin, A. G. Jansen, C. Nguyen, B. Brar, M. Thomas, and H. Kroemer, InAs-AlSb quantum wells in tilted magnetic fields, Phys. Rev. B 61, 13045 (2000).

[36] K. F. Yang, H. W. Liu, T. D. Mishima, M. B. Santos, K. Nagase, and Y. Hirayama, Nonlinear magnetic field dependence of spin polarization in high-density two-dimensional electron systems, New J. Phys. 13, 083010 (2011).

[37] J. C. Chokomakoua, N. Goel, S. J. Chung, M. B. Santos, J. L. Hicks, M. B. Johnson, and S. Q. Murphy, Ising quantum Hall ferromagnetism in InSb-based two-dimensional electronic systems, Phys. Rev. B 69, 235315 (2004).

[38] S. Koch, R. J. Haug, K. v. Klitzing, and M. Razeghi, Suppression of the Landau-level coincidence: A phase transition in tilted magnetic fields, Phys. Rev. B 47, 4048(R) (1993).
[39] E. P. De Poortere, E. Tutuc, S. J. Papadakis, and M. Shayegan, Resistance spikes at transitions between quantum Hall ferromagnets, Science 290, 1546 (2000).

[40] E. P. De Poortere, E. Tutuc, and M. Shayegan, Critical Resistance in the AlAs Quantum Hall Ferromagnet, Phys. Rev. Lett. 91, 216802 (2003).

[41] H. P. Wei, A. M. Chang, D. C. Tsui, and M. Razeghi, Temperature dependence of the quantized Hall effect, Phys. Rev. B 32, 7016(R) (1985).

[42] J. Zhu, H. L. Stormer, L. N. Pfeiffer, K. W. Baldwin, and K. W. West, Spin Susceptibility of an Ultra-Low-Density TwoDimensional Electron System, Phys. Rev. Lett. 90, 056805 (2003).

[43] B. Nedniyom, R. J. Nicholas, M. T. Emeny, L. Buckle, A. M. Gilbertson, P. D. Buckle, and T. Ashley, Giant enhanced $g$-factors in an InSb two-dimensional gas, Phys. Rev. B 80, 125328 (2009).

[44] W. Desrat, F. Giazotto, V. Pellegrini, M. Governale, F. Beltram, F. Capotondi, G. Biasiol, and L. Sorba, Anticrossings of spin-split Landau levels in an InAs two-dimensional electron gas with spin-orbit coupling, Phys. Rev. B 71, 153314 (2005).

[45] K. J. Goldammer, S. J. Chung, W. K. Liu, M. B. Santos, J. L. Hicks, S. Raymond, and S. Q. Murphy, High-mobility electron systems in remotely-doped InSb quantum wells, J. Cryst. Growth 201-202, 753 (1999).

[46] D. C. Tsui, H. L. Stormer, and A. C. Gossard, Two-Dimensional Magnetotransport in the Extreme Quantum Limit, Phys. Rev. Lett. 48, 1559 (1982).

[47] S. F. Nelson, K. Ismail, J. J. Nocera, F. F. Fang, E. E. Mendez, J. O. Chu, and B. S. Meyerson, Observation of the fractional quantum Hall effect in $\mathrm{Si} / \mathrm{SiGe}$ heterostructures, Appl. Phys. Lett. 61, 64 (1992).

[48] C. R. Dean, A. F. Young, P. Cadden-Zimansky, L. Wang, H. Ren, K. Watanabe, T. Taniguchi, P. Kim, J. Hone, and K. L. Shepard, Multicomponent fractional quantum Hall effect in graphene, Nat. Phys. 7, 693 (2011). 